\title{
Enterotoxin-Nachweis in Kulturfiltraten von Staphylococcus aureus mittels ELISA
}

A. Pickert, H. Mörk, H. Werner

\begin{abstract}
Zusammenfassung:
185 Staphylococcus-aureus-Isolate wurden mit einem kommerziell erhältlichen ELISA auf Enterotoxinproduktion Typ A-D überprüft. Die Stämme waren Isolate aus verschiedenen klinischen Materialien sowie Abstrichen von Kücheneinrichtungen und Lebensmitteln. In Urinproben reagierten 57\% (16/28), in Wundabstrichen und Stuhlproben 24\% (10/41; 6/25), in Hautabstrichen 21\% (6/28), in Eiter von Panaritien 16\% (4/25) positiv. Unter 38 Isolaten von Abstrichen des Wirtschaftskontrolldienstes reagierten 13\% (5/38) positiv. Enterotoxin $A, B$ und $C$ waren annähernd gleich häufig nachweisbar, Enterotoxin $D$ wurde seltener gefunden. Der ELISA erwies sich als sicher und praktikabel in der routinemäßigen Anwendung; seine Empfindlichkeit konnte durch externe und vom Hersteller gelieferte Positivkontrollen mit 0,1-0,5 ng für die Enterotoxine $A-B$ und 0,5-1 $\mu \mathrm{g}$ für Enterotoxin D bestätigt werden. Enterotoxin-produzierende Staphylococcus-aureus-Stämme waren im Stuhl von Patienten mit Gastroenteritis nicht häufiger zu finden als in anderen klinischen Untersuchungsmaterialien. Der Nachweis einer Enterotoxinproduktion in vitro bei Isolaten aus Stuhl erscheint daher von fraglicher pathogenetischer Relevanz für die Diagnostik einer Gastroenteritis zu sein.
\end{abstract}

Schlüsselwörter:

Staphylococcus aureus - Enterotoxine

Summary:

185 isolates of Staphylococcus aureus were analyzed for enterotoxin production. A commercially available ELISA for enterotoxins type $A-D$ was used. The strains were isolated from different materials. The percentage of enterotoxin-producing strains was $57(16 / 28=n$ positive/n tested) among isolates from urine, 24 (10/ 41 and 6/25 respectively) from wounds and faeces, 21 (6/28) from skin swabs, 16 (4/25) from paronychia and 13 (5/38) from food and kitchen-fittings. Enterotoxins type $A-C$ were frequently detected, enterotoxin $D$ was infrequently found. The ELISA was practicable under conditions of a bacteriological routine laboratory. The sensitivity was estimated being. $0.1-0.5 \mathrm{ng}$ for enterotoxin $A-C$ and being $0.5-1 \mu g$ for enterotoxin $D$. The percentage of enterotoxin-producers among isolates from faeces of patients with gastroenteritis was equal to or even lower than that found in other clinical materials. This could mean that identifying enterotoxinproducing staphylococci in faeces, is not helpful in diagnosing the etiological agent of gastroenteritis.

Keywords:

Staphylococcus aureus - Enterotoxins

\section{Einleitung}

Die Staphylokokken-Enterotoxin-Gastroenteritis imponiert klinisch durch ihr Auftreten als lokale Epidemie mit einer kurzen Inkubationszeit (kleiner als $6 \mathrm{Std}$.) (12); in der Regel gehören die Patienten einer Eßgemeinschaft an. Zur Sicherung der Diagnose im Labor ist der Nachweis der Staphylokokken-Enterotoxine in verdächtigten Lebensmitteln, Erbróchenem oder Kulturfiltraten zu fordern. In praxi mißlingt dies fast immer, da die Lebensmittel nicht mehr verfügbar sind, Erbrochenes nicht zur Untersuchung gelangt und der Nachweis von Staphylococcus aureus in leicht zugänglichen Untersuchungsmaterialien wie Stuhl oder Abstrichen von Kücheneinrichtungen nicht beweisend ist (3).

Zum Enterotoxinnachweis sind zahlreiche Methoden entwickelt worden. Präzipitationsreaktionen als AgargelDoppeldiffusion [OSP-Methode $(6,11)$ ] oder auf dem Objektträger [Microslide-Test $(4,14)$ ] gelten als Standardverfahren; entsprechende Reagenzien sind im Han- del erhältlich; die Methoden müssen jedoch im Labor selbst etabliert werden. Der Nachteil liegt in der geringen Empfindlichkeit. Kompetitive Proteinbindungsanalysen nach dem RIA- oder ELISA-Prinzip und ein Latex-Test (SET-RPLA, Oxoid, Wesel) sind mehrfach beschrieben worden. Reagenzien für ELISA- und Latex-Test sind als gebrauchsfertige Testpackungen von Diagnostika-Herstellern erhältlich. Nachteil ist die gegenüber den Präzipitationsverfahren geringere Spezifität. In der Arbeitsgruppe von. Fey et al. (2) wurde ein nach dem SandwichVerfahren arbeitender ELISA zum Nachweis der Enterotoxine A, B, C und D entwickelt, der den Anforderungen eines Routineverfahrens hinsichtlich Stabilität und Reproduzierbarkeit entspricht. Dieser Test ist kommerziell erhältlich. Von Lenz et al. (5) konnte unter Verwendung der Reagenzien von Fey et al. (2) gezeigt werden, daß dieser ELISA empfindlicher, besser reproduzierbar und schneller als der Microslide-Test ist.

Aktueller Anlaß für unsere Untersuchung war eine Massenerkrankung in Tübingen. Bei einigen Erkrankten konn- 
ten wir Staphylococcus aureus im Stuhl nachweisen. Es erhob sich die Frage der pathogenetischen Relevanz dieses Befunds. Das Enterotoxinbildungsvermögen und der Phagenlysotyp der Stämme wurden auswärtig untersucht (Lenz, W.: Staphylokokken-Zentrale am Institut für Medizinische Mikrobiologie und Immunologie der Universität Bonn). Die untersuchten Stämme gehörten alle verschiedenen Lysotypen an; mit einer Ausnahme waren alle Stämme keine Enterotoxinproduzenten. Dies veranlaßte uns zu prüfen, inwieweit aus Stuhl isolierte Stämme von Staphylococcus aureus im Vergleich zu Isolaten aus anderen Materialien Enterotoxine produzieren.

\section{Material und Methoden}

Zur Isolierung von Staphylococcus aureus aus Stuhl wurde eine erbsengroße Stuhlprobe in Kochsalz-FleischBouillon (Oxoid CM 94) eingebracht und 18-24 Std. bei $37^{\circ} \mathrm{C}$ bebrütet. Die Bouillon wurde anschiließend auf Columbia-CNA-Blutagar (Oxoid CM 331, SR 70) und Mannit-Kochsalz-Agar (Oxoid CM 85) 3fach fraktioniert ausgestrichen. Nach weiterer 18-24stündiger Bebrütung bei $37^{\circ} \mathrm{C}$ wurden verdächtige Kolonien abgeimpft und mit biochemischen Kriterien (s.u.) identifiziert. Zur Isolierung von Staphylococcus aureus aus Urinproben, Abstrichen und Eiter wurden mikrobiologische Standardverfahren angewendet. Die Bewertung der Signifikanz eines Bakteriennachweises im Urin (Keimzahl, Bedeutung von Mischkulturen, Hemmstoffnachweis) erfolgte gemäß den Vorschriften der DGHM (1). Die biochemische Identifikation der Spezies Staphylococcus aureus erfolgte durch Plasmakoagulase, DNAse, Mannitspaltung und OF-Test.

Der Nachweis der Staphylococcus-aureus-Enterotoxine erfolgte nach der Vorschrift des Reagenzien-Herstellers (Labor Dr. W. Bommeli, Länggasstr. 7, CH 3012 Bern). Alle erforderlichen Reagenzien für den ELISA wurden vom Hersteller mitgeliefert.

Testprinzip: Mit Antikörpern gegen Staphylokokken-Enterotoxin beschichtete Kugeln werden zum Kulturfiltrat gegeben; Enterotoxine, falls vorhanden, binden an die Kugeln. Die gebundenen Enterotoxine werden mit einem zweiten Antikörper, der Enzym-markiert ist, gekoppelt. Die Enterotoxinbindung an die Kugeln wird durch Substratzugabe sichtbar gemacht. Der Enterotoxinnachweis wurde im Kulturfiltrat einer Subkultur durchgeführt. 2-3 typische Kolonien wurden in Nährbouillon (Nährbouillon Nr. 2; CM 67, Oxoid) überimpft und bei $37^{\circ} \mathrm{C} 18-24$ Std. bebrütet. Die bewachsene Nährbouillon wurde filtriert (Einmalfilter, Porengröße: $0,45 \mu$, Millipore). Pro Enterotoxin-Ansatz wurden $400 \mu$ Kulturfiltrat eingesetzt. Die Auswertung erfolgte in . Flachboden-Mikrotiterplatten (Greiner, Nürtingen). Die Extinktion bei $405 \mathrm{~nm}$ wurde in einem ELISA-Photometer für Mikrotiterplatten gemessen (Virion, Würzburg).

Die Bewertung der Meßergebnisse (Optische Dichte) erfolgte nach folgendem Verfahren: Aus den Werten der Negativkontrollen für die Enterotoxine A, B und C eines Tagesansatzes wurde das $99 ; 7 \%$. Vertrauensintervall des Mitteiwerts errechnet (Mittelwert \pm 3 fache Standardabweichung). Für jede gemessene Einzelprobe wurde ein Quotient berechnet:
Falls der Quotient $\geqslant 2,0$ war, wurde die Probe für das entsprechende Enterotoxin als positiv gewertet. Für die Bestimmung des Enterotoxin D mußte ein anderes Verfahren gewählt werden, da die Negativkontrollen, bedingt durch die höheren Proteinkonzentrationen (W. Bommeli, persönliche Mitteilung), durchweg um 2-3 Größenordnungen höher lagen als die für die übrigen Enterotoxine. Aus insgesamt 28 gemessenen Negativkontrollen für Enterotoxin D wurde ein Mittelwert + 3fache Standardabweichung von 0,095 ermittelt. Alle Proben, deren Extinktionen größer waren, wurden als positiv gewertet. Als Positivkontrollen wurde pro Tagesansatz einmal ein kompletter Satz Enterotoxine A, B, C und D mitbestimmt (erhältlich bei Serva, Heidelberg oder Dr. Bommeli, Bern). Die Empfindlichkeit des ELISA wurde durch geometrische Reihenverdünnung mit ca. 0,1-0,5 ng für die Enterotoxine $A, B, C$ und ca. $0,5-1 \mu \mathrm{g}$ für Enterotoxin $D$ pro Ansatz bestimmt.

\section{Ergebnisse}

Vom April bis November 1985 wurden 210 Stuhlproben von Patienten mit akuter Gastroenteritis (klinische Diagnose) auf Enterotoxin-produzierende Staphylokokken untersucht. Aus 27 Stuhlproben (12,8\%) konnte Staphylococcus aureus als einziger fakultativ pathogener Keim angezüchtet werden (ausgeschlossen wurden Proben, in denen Salmonellen, Shigellen, Yersinien oder Campylobacter, bei Säuglingen auch Rotaviren, nachgewiesen worden waren). Die 27 Isolate $(=100 \%)$ verteilten sich wie folgt auf die verschiedenen Nährmedien: 20 Stämme (74\%) wurden sowohl auf CNA-Agar als auch auf Mannit-Agar isoliert, 6 Stämme (22\%) wurden ausschließlich auf CNA-Agar isoliert, und 1 Stamm wurde nur auf Mannit-Agar isoliert (4\%). Weitere 14 Staphylococcus-aureus-Stämme aus Stuhlproben wurden uns von anderen Untersuchern zur Prüfung auf Enterotoxinproduktion überlassen. Enterotoxine konnten im Kulturfiltrat bei 10 der 41 isolierten Stämme nachgewiesen werden (24\% der Isolate, vgl. Tab. 1).

Neben Stuhlproben wurden andere klinische Materialien wie Urin, Wundabstriche, Hautabstriche und Eiter untersucht. Bei 28 Isolaten aus Urin von Patienten mit signifikanter Bakteriurie (Keimzahl: $>10^{5} / \mathrm{ml}$ ) konnte $16 \mathrm{mal}$ (57\%) Enterotoxinproduktion nachgewiesen werden (vgl. Tab.1). Bei 25 Staphylococcus-aureus-Stämmen, isoliert aus Wundabstrichen (Herkunft: Orthopädische Universitätsklinik und Berufsgenossenschaftliche Unfallklinik), konnte 6mal Enterotoxinproduktion nachgewiesen werden (24\%, vgl. Tab. 1). Bei 28 Stämmen aus ekzematös oder entzündlich veränderter Haut (Herkunft: Universitäts-Hautklinik) reagierten 6 positiv (21\%, vgl. Tab. 1). Aus Eiter bzw. Wundabstrichen von chirurgisch versorgten Panaritien und Abszessen (Herkunft: Chirurgische Universitätsklinik und Berufsgenossenschaftliche Unfallklinik) konnten unter 25 Staphylococcus-aureusStämmen nur 4 positiv reagierende isoliert werden $(16 \%$, vgl. Tab. 1).

38 Staphylococcus-aureus-Isolate aus nicht-menschlichen Untersuchungsmaterialien (Kontrollabstriche des Wirtschaftskontrolldienstes von Lebensmitteln und Kü-

Extinktion [Probe]

Extinktion [Mittelwert Negativkontrollen + 3fache Standardabweichung] 
Tab. 1: Untersuchung der Enterotoxinproduktion im Kulturfiltrat mittels ELISA bei verschiedenen Isolaten von Staphylococcus aureus (Abkürzungen: $n=$ Absolutwerte, $W K D=$ Wirtschaftskontrolldienst; Abstriche von Kücheneinrichtungen)

Staphylococcus-aureus-isolate

\begin{tabular}{|c|c|c|c|c|c|c|c|c|c|}
\hline \multirow{3}{*}{ Stuhlproben } & \multirow{2}{*}{$\begin{array}{l}\text { gesamt } \\
n\end{array}$} & \multicolumn{2}{|c|}{$\begin{array}{l}\text { Enterotoxin- } \\
\text { positiv }\end{array}$} & \multicolumn{6}{|c|}{ Enterotoxin-Typ } \\
\hline & & $n$ & $\%$ & A & B & c & D & $A+B$ & $B+D$ \\
\hline & 41 & 10 & 24 & 3 & 1 & 4 & 2 & - & - \\
\hline $\begin{array}{l}\text { Urinproben } \\
\text { Wundabstriche } \\
\text { Hautabstriche } \\
\text { Eiter }\end{array}$ & $\begin{array}{l}28 \\
25 \\
28 \\
25\end{array}$ & $\begin{array}{r}16 \\
6 \\
6 \\
4\end{array}$ & $\begin{array}{l}57 \\
24 \\
21 \\
16\end{array}$ & $\begin{array}{l}4 \\
1 \\
2 \\
2\end{array}$ & $\begin{array}{r}6 \\
2 \\
1 \\
-\end{array}$ & $\begin{array}{l}2 \\
2 \\
- \\
-\end{array}$ & $\begin{array}{l}- \\
1 \\
1 \\
2\end{array}$ & $\begin{array}{l}3 \\
\frac{1}{1} \\
-\end{array}$ & $\frac{1}{1}$ \\
\hline WKD-Abstriche & 38 & 5 & 13 & 2 & - & - & 3 & - & - \\
\hline Summe & 185 & 47 & 25,4 & 14 & 10 & 8 & 9 & 4 & 2 \\
\hline
\end{tabular}

cheneinrichtungen) wurden uns freundlicherweise von Herrn Dr. Riethmüller aus dem Medizinischen Landesuntersuchungsamt Stuttgart (Direktor: Prof. Dr. W. Steuer) zur Verfügung gestellt. Unter diesen Isolaten konnten nur 5 Enterotoxin-Produzenten nachgewiesen werden (13\%, vgl. Tab. 1).

\section{Diskussion}

Mit dem ELISA der Fa. Bommeli, der wissenschaftlich von Fey et al. (2) ausgearbeitet wurde, steht ein erprobtes Testsystem zur Bestimmung der 4 Enterotoxine A-D von Staphylococcus aureus zur Verfügung. Die Durchführung des ELISA erwies sich als sicher und praktikabel in der täglichen Anwendung. Die Auswertung mußte für die Enterotoxine A, B, C einerseits und Enterotoxin D andererseits getrennt vorgenommen werden, da der Enterotoxin-D-ELISA zu unspezifischen Reaktionen neigte. Dies erforderte die Untersuchung einer größeren Gruppe von negativen Kontrollproben für Enterotoxin D. Das zur Gewinnung des Kulturfiltrats verwendete Nährmedium kann einen deutlichen Effekt auf den ELISA haben. Wir hatten in einer Versuchsserie, in der statt der beschriebenen einfachen Nährbouillon Leberbouillon genommen wurde, in sämtlichen ELISA-Proben einschließlich der Negativkontrollen stark positive Ergebnisse, so daß die Resultate nicht interpretierbar waren.

Zur Anreicherung von Staphylococcus aureus aus polymikrobiell besiedelten Materialien wie zum Beispiel Stuhl kann aufgrund unserer Untersuchungen eine Kombination aus einer Flüssiganreicherung in Kochsalz-FleischBouillon und der CNA-Platte, die das Wachstum von gramnegativen Bakterien unterdrückt, empfohlen werden. Der Mannit-Kochsalz-Agar, ein Standardnährmedium zur Isolierung von Staphylococcus aureus, war der CNA-Platte bei der Untersuchung von Stuhlproben deutlich unterlegen. Trotz optimierter Anzüchtungsbedingungen konnten wir in 210 untersuchten Stuhlproben von Patienten mit Gastroenteritis nur 27 (12,8\%) Staphylococcus-aureus-Stämme nachweisen. Von diesen erwiesen sich nur 5 als Enterotoxin-Produzenten (2,4\%). Falls den Erkrankungen eine Enterotoxin-Vergiftung zugrunde lag, muß man annehmen, daß die Erreger nur in geringer Menge aufgenommen wurden, oder die Magen-DarmPassage nicht überlebten oder daß das Enterotoxinbildungsvermögen in vitro verloren ging. Der Wirkmechanismus der hitzestabilen Enterotoxine führt durch allei- nige Aufnahme des Toxins mit der Nahrung zu Krankheitssymptomen; die Erreger selbst können z. B. durch Kochen abgetötet sein. Die wenigen Enterotoxin-positiven Befunde in unserer Untersuchung lassen es darüber hinaus fraglich erscheinen, ob dem Nachweis von Enterotoxin-produzierenden Staphylokokken im Stuhl angesichts der Häufigkeit asymptomatischer Keimträger überhaupt eine krankheitsdiagnostische Relevanz zukommt Neben der Enterotoxinproduktion existieren noch andere Mechanismen, die eine Darmerkrankung durch Staphylococcus aureus bewirken können. Eine enteroinvasive Infektion kann zu profusen Durchfällen bei Patienten unter Antibiotika-Therapie führen (3). Durchfallserkrankungen können auch durch toxische Wirkungen der lipolytischen Aktivität von Staphylococcus aureus entstehen (13).

Überraschend an den übrigen von uns untersuchten klinischen Materialien war die relativ hohe Zahl Enterotoxinpositiver Staphylococcus-aureus-Isolate im Urin und im Vergleich hierzu die relativ niedrige Zahl in Abszessen und Panaritien. Wenn man annimmt, daß die Enterotoxinproduktion ein Pathogenitätskriterium von Staphylococcus aureus ist, so könnte die relative Häufigkeit des Enterotoxinnachweises mit der Signifikanz des Erregernachweises im entsprechenden Untersuchungsmaterial korrelieren. Für korrekt abgenommene Urinproben gelten strenge Kriterien der Bewertung eines Bakteriennachweises (1). Beim Nachweis von Staphylococcus aureus in hoher Keimzahl als Monokultur im Urin handelte es sich demnach mit großer Wahrscheinlichkeit um den Erreger eines Infektionsprozesses. Angesichts einer Keimträgerquote von 30 bis $40 \%$ unter der gesunden Bevölkerung muß bei allen Materialien auch eine Kontamination durch saprophytäre Stämme der Haut in Erwägung gezogen werden.

Die Gesamtzahl an Staphylokokken-Enterotoxin-positiven Befunden in unserer Studie entspricht den Ergebnissen anderer Autoren. Richter et al. (10) fanden mit dem Microslide-Test in Isolaten aus Patienten 28\% Enterotoxin A-, B-, C- oder D-produzierende Stämme, wobei die einzelnen Toxine annähernd gleich häufig vertreten waren. In einer Kontrollgruppe von Gesunden (Nasenabstriche) wurde jedoch in $42 \%$ der Isolate Enterotoxinproduktion nachgewiesen (davon alleine 27,9\% Enterotoxin-A-produzierende Stämme). Insgesamt waren Enterotoxin-Produzenten sehr viel häufiger bei Isolaten aus menschlichem als bei Isolaten aus tierischem Material. Mochmann et al. (8) untersuchten verschiedene Staphylococcus-aureus-Isolate von Patienten (Wundabstriche, Nasen-Rachenabstriche, Eiter bei Mastitis); in ca. $30 \%$ 
ließ sich mit dem Microslide-Test Enterotoxinproduktion nachweisen. Demgegenüber ließen sich in Abstrichen, die im Zusammenhang mit Lebensmittelvergiftungen von verschiedenen Küchenmaterialien und -gegenständen angelegt worden waren, nur in 12,5\% Enterotoxinproduktion nachweisen, dies entspricht unseren Ergebnissen. Olsvik et al. (9) untersuchten mit dem ELISA Staphylococcus-aureus-Isolate verschiedener Herkunft; $42 \%$ der menschlichen Isolate reagierten positiv. Malconian et al. (7) untersuchten mit dem Microslide-Test die Enterotoxinproduktion $(A, B, C)$ von Staphylococcus-aureusIsolaten verschiedener Körperregionen (Haut, Respirationstrakt, Liquor, Urin, Blut, Vagina); zwischen $37 \%$ (Haut) und $50 \%$ (Liquor) der Stämme erwiesen sich als positiv. Mauff et al. (6) untersuchten mit der OSP-Methode 208 Isolate aus klinischem Material. 88 Stämme produzierten ein oder mehrere Enterotoxine (42\%). Stämme, die bei systemischen Infektionen (Sepsis, Meningitis) isoliert wurden, zeigten in 57\% Enterotoxinproduktion. Die kurze Übersicht zeigt, daß je nach Autoren und Methode die Rate positiver Befunde schwankt. Unter den einzelnen Enterotoxinen läßt sich aus menschlichem Material am häufigsten Enterotoxin A nachweisen; dies wird auch durch unsere Ergebnisse bestätigt.

Unsere Untersuchungen zeigten, daß sich in Staphylococcus-aureus-Isolaten aus Stuhlproben nicht häufiger Enterotoxine nachweisen lassen als in Isolaten von anderen klinisch relevanten Körperregionen. Eine klinische $\mathrm{Be}$ wertung des Enterotoxinnachweises im Kulturfiltrat von Staphylococcus-aureus-Isolaten aus Stuhl ist daher nur in den Fällen möglich, in denen zusätzliche Hinweise durch die Anamnese und den epidemiologischen Verlauf für eine Staphylokokken-Enterotoxin-Erkrankung sprechen.
Schrifttum:

1. BURKHARDT, F., BOLTZE, H.-J., ULLMANN, U.: Die bakteriologische Diagnose der Harnwegsinfektion. F. BURKHARDT (Hrsg.): Verfahrensrichtlinien für die mikrobiologische Diagnostik. G. Fischer, Stuttgart, 1981.

2. FEY, H., PFISTER, H., RÜEGG, O.: Comparative evaluation of different enzymelinked immunosorbent assay systems for the detection of staphylococcal enterotoxins

A, B. C, and D. J. Clin. Microbiol. 19, 34-38 (1984).
3. KIST, M., ALEKSIC, S., BOCKEMUHL. J., DAHN, R., PRITZBUER, V., MÜLLER, 3. KIST. M., ALEKSIC, S., BOCKEMUHL. J.. DAHN, R., PRITZBUER, V., MÜLLER,
H. E., KUNKEL. J., HOFFMAN, K.: Die bakteriologische Diagnostik enteraler Infektionen. F. BURKHARDT (Hrsg.): Verfahrensrichtlinien für die mikrobiologische Diagnostik. G. Fischer, Stuttgart, 1981.

4. LANGNER, H. J.: An apparatus for the microslide immunodiffusion. Yokohama Med. Bull. 23, 89-94 (1973).

5. LENZ, W. THELEN, R. PICKENHAHN P. BRANDIS, $H$. Nachweis von Enteroto $x$ in in Kulturüberständen von Staphylococcus aurous mit dem ELISA.Test und dem Microslide-Test. Zbl. Bakt. Hyg., I.Abt. Orig. A 253, 466-475 (1983).

6. MAUFF, G., RÖHRIG, I., ERNZER, U., BERGDOLL; M., PULVERER, G.: Enterotoxigenicity of Staphylococcus aureus strains from clinical isolates. Eur. J. Clin. Microbiol. $2,321-326(1983)$

7. MELCONIAN, A. K., BRUN, Y., FLEURETTE, J.: Enterotoxin production, phage typing and serotyping of Staphylococcus aureus strains isolated from clinical materials and food. J. Hyg. Camb. 91, 235-242 (1983).

8. MOCHMANN, H., AKATOV, A. K., KHATENEVER, M. L., RICHTER, U., KUSCHKO, 1. W., KARSCH, W.: Studies on enterotoxin production by strains of Staphylococcus of different origin obtained from USSR. J. ILJASZEWICZ (ed.): Staphylococci and Staphylococcal Infections. Zbl. Bakt. Suppl. 10, Gustav Fischer Verlag. Stuttgan, New York, 1981.

9. OLSVIK, O., BERDAL, B. P., FOSSUM, K., OMLAND, T.: Enterotoxin production by Staphylococcus aureus related to the origin of the strains. Acta path. microbiol. scand. Sect. B 89, 423-426 (1981)

10. RICHTER, U., WITTE, W., HUMMEL, R., KARSCH, W., MOCHMANN, H.: Enterotoxin production and host specific variety by Staphylococcus aureus strains. J. ILJASZEWICZ (ed.): Staphylococci and Staphylococcal Infections. Zbl. Bakt. Suppl. 10. Gustav Fischer Verlag. Stuttgart, New York, 1981.

11. ROBBINS, R., GOULD. S., BERGDOLL, M.: Detecting the enterotoxigenicity of Staphylococcus aureus strains. Appl. Mikrobiol. 28, 946-950 (1974).

12. SINELL, H. J.: Bedeutung von Staphylokokken-Enterotoxinen in Lebensmitteln Med. Klin. 71, 1165-1171 (1976)

13. SOUREK, J., NEUBAUER, M., MILACEK, V., BERGDOLL, M. S., SOUCEK, A. Relation of enterotoxin production and lipolytic activity in Staphylococcus aureus strains isolated from acute diarrhoel diseases to clinical course of illnes. J. Hyg. Epidemiol. Microbiol. Immunol. 28, 279-286 (1984).

14. WADSWORTH. C.: A slide microtechnique for the analysis of immune precipitates in gel. Int. Arch. Allergy. 10, 355-360 (1955).

Anschrift der Verfasser:

Dr. med. Dr. rer. nat. A. Pickert*

Abt. Medizinische Mikrobiologie

Direktor: Prof. Dr. H. Werner

Hygiene-Institut der Universität

Silcherstraße 7

7400 Tübingen

- Neue Korrespondenzadresse:

Medizinische Klinik, Abt. IV

Direktor: Prof. Dr. med. M. Eggstein

Otfried-Müller-Straße

7400 Tübingen

Mörck angefertigten Dissertation. 